\title{
Using Systems Thinking to Exponentially Increase Impact
}

\author{
Andy E. Williams, Nobeah Foundation, Nairobi, Kenya
}

\begin{abstract}
This paper explores how Human-Centric Functional Modeling might provide a method of systems thinking that in combination with models of Artificial General Intelligence and General Collective Intelligence developed using the approach, creates the opportunity to exponentially increase impact on targeted outcomes of collective activities, including research in a wide variety of disciplines as well as activities involved in addressing the various existential challenges facing mankind. Whether exponentially increasing the speed and scale of progress in research disciplines such as physics or medicine, or whether exponentially increasing capacity to solve existential challenges such as poverty or climate change, this paper explores why gaining the capacity to reliably solve such challenges might require this exponential increase in general problem-solving ability, why this exponential increase in ability might be reliably achievable through this approach, and why solving our most existential challenges might be reliably unachievable otherwise.
\end{abstract}

\section{Introduction: What is Human-Centric Functional Modeling?}

In Human-Centric Functional Modeling living systems such as the human organism are modeled as having a set of human-observable behaviours (functions). When all the states accessible through these functions occur within a given domain of behaviour (i.e. all states belong to some common category), these states form a "functional state space", which the system acting in that domain moves through. All the states accessible through those functions (i.e. all the states in the "functional state space") then reflect all possible behaviors of the system. This functional state space can potentially be virtual and abstract, such as in the case of the cognitive system which can be represented as moving through a space of concepts (a "conceptual space"), or it can be physical, such as the sensory-motor space the body can be represented as moving through. Functional state spaces can be defined for every system within or outside the human organism that can be observed by the human organism, including the body, emotions, mind, and consciousness. If each functional state space represents all possible behaviors of the system that can be perceived by a human, then all models for that system that are defined by any theory must define behaviors that are confined to that space, at least wherever those models represent actual functionality that can be executed within the capacity of the system (i.e. wherever those models are real).

When applied to understanding human systems such as cognition, a human-centric approach allows systems to be understood through first person observation (observations that can be validated within the individual's observation of their own awareness), as opposed to observations requiring third party validation (i.e. measurements made by external tools or assessing validity in terms of consistency with some theory defined by some third party). In understanding human systems like cognition for which many of the functions cannot be externally measured, a first person approach is essential.

\section{Background: The Big Picture Regarding Systems Thinking}

In order to be maximally generalizable to concepts and reasoning in each individual's conceptual space, any method of systems thinking must minimize the requirements to understand and apply systems thinking. One of the reasons that systems research has not spread more in the past 50 years might be the 
lack of a systems approach that is useful in solving a sufficiently broad set of problems and that can be spread sufficiently widely. It has been said that "there is no commonly accepted definition of what a Complex System is" [1]. If we could all see the world in terms of the same one system, we could maximize our ability to spread the benefit of systems thinking everywhere. The only system that every human being has in common is the human organism itself.

\section{$3 \quad$ Functional State Spaces}

Human-Centric Functional Modeling represents this human system as well as all of this existence that can be perceived by this human system in terms of mathematical spaces (functional state spaces) that are innate to the way every single human being on earth perceives. For the first time in history these mathematical spaces are hypothesized to allow us to turn concepts like complexity or intelligence into mathematical equations that we can validate within our own first person awareness of our own experience. This approach has been used to develop models of individual, artificial [2], or collective cognition [3] that are predicted to exponentially increase our ability to navigate complexity in order to solve problems [5]. This approach might also potentially be used to represent and increase our ability to understand any system that can be conceptualized. The reason is that conceptual space is represented by a graph containing a network of nodes representing concepts and connected by edges representing reasoning or understanding processes. A graph of nodes that interact through edges describes every region in the physical universe that interacts through forces. It has been claimed that "all Complex Systems can be represented and described as networks" [7]. If so then Human-Centric Functional Modeling can potentially also be used to represent the physical universe in order to leverage the exponentially greater general problem-solving ability of AGI or GCI as increasing AGI and GCI functionality becomes available, in order to radically increase our ability to solve problems in physics or mathematics [6]. In the same way, HCFM might also be used to represent social or economic systems in order to radically increase our capacity to achieve social or economic outcomes through complex projects. Or HCFM might be used to represent computer systems in order to radically increase our capacity to achieve computing goals [8]. The applications might be endless.

From the Human-Centric Functional Modeling perspective systems can potentially be represented not just as networks defined in terms of the theoretical components of those systems, but in terms of the way those systems are perceived. One might choose to represent a curtain covering a window in terms of nodes of some size representing a pixel rather than in terms of nodes representing imperceptible physical entities such as molecules, since computing motion or other outcomes in terms of molecules might make problems intractable. Those pixels might be smaller or larger depending on factors like the level of detail in a particular region of the fabric, and the light levels at each time of day which determines the resolution of our eyes. The fact that humans innately define systems in terms of their individual perception and that this perception might vary is demonstrated in a recent paper [9] in which nodes of potential networks have been perceived in a wide variety of systems, including the nervous system, in the immune system, in biological ecosystems, and even the climate, where those nodes could potentially have been defined in multiple ways.

Rather than being completely new, Human-Centric Functional Modeling leverages the various many thousand year old existential traditions for first person observation, such as the yogic tradition, which suggest we can only understand this existence through its interaction with this human system, and that by sufficiently increasing our awareness of this human system and what it perceives, we can become aware of this entire existence. Human-Centric Functional Modeling turns this perception into mathematics so that it can more readily be communicated and so insight can more reliably be achieved. 
In essence Human-Centric Functional Modeling is a model of computation for these existential traditions.

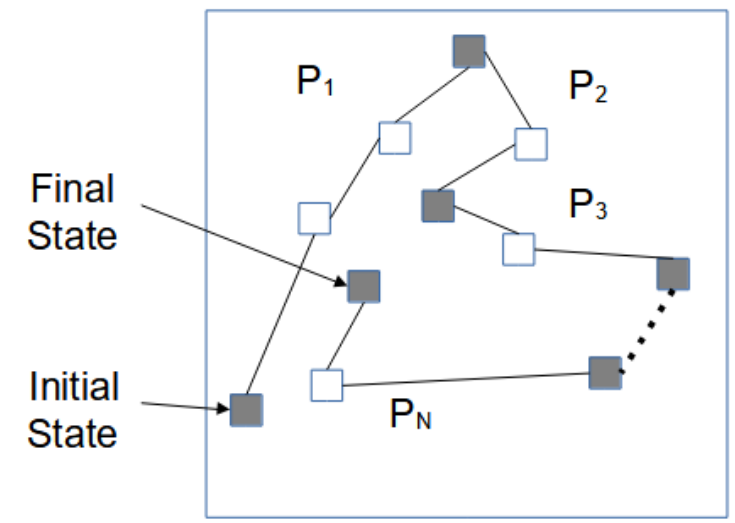

Figure 1: In Human-Centric Functional Modeling systems are represented as moving through a functional state space.

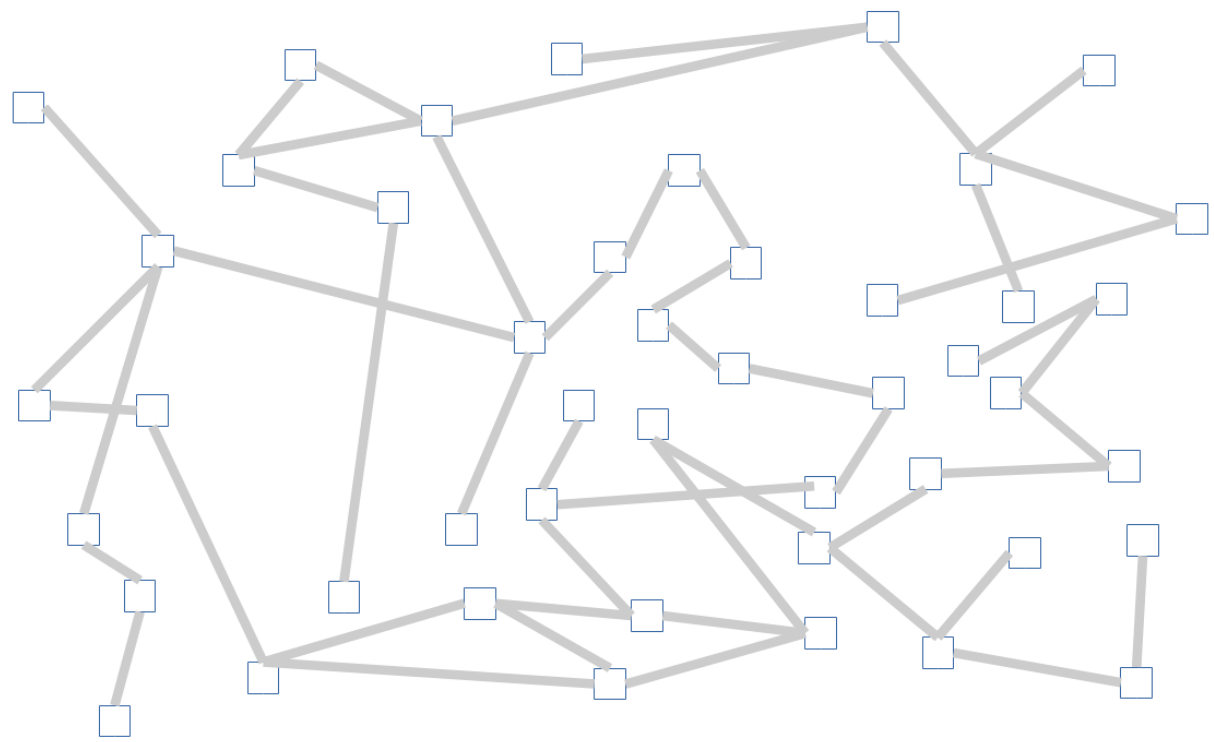

Figure 2: Any functional state space is represented by a network of nodes representing the functional states, connected by edges representing the processes by which the system transitions from one functional state to another. 


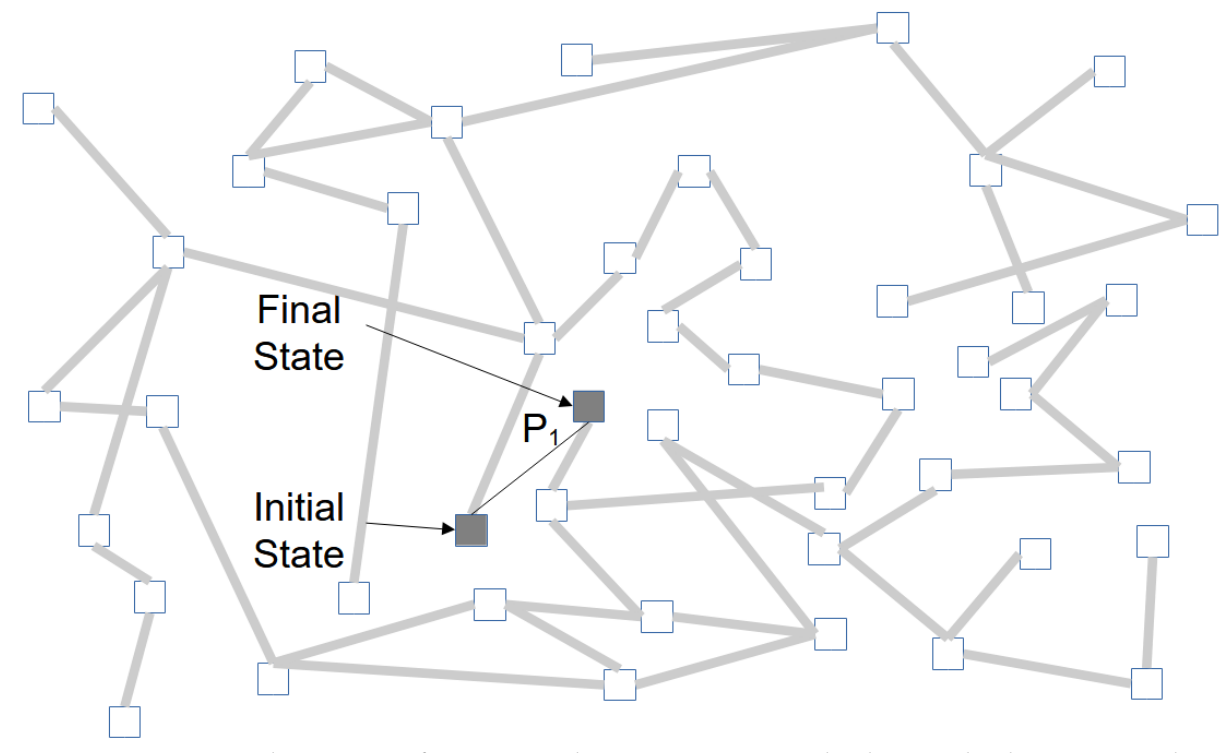

Figure 3: An interaction between functional states is a path through the network of functional states. In this sense, within conceptual space (the functional state space of the cognitive system) a reasoning process used by the cognitive system to navigate from one concept to another is an interaction between those two concepts. In functional state space the initial state and final state are nodes of the network that interact through the edges in the network representing the functional state space. A direct interaction represents type 1 (intuitive) reasoning in conceptual space.

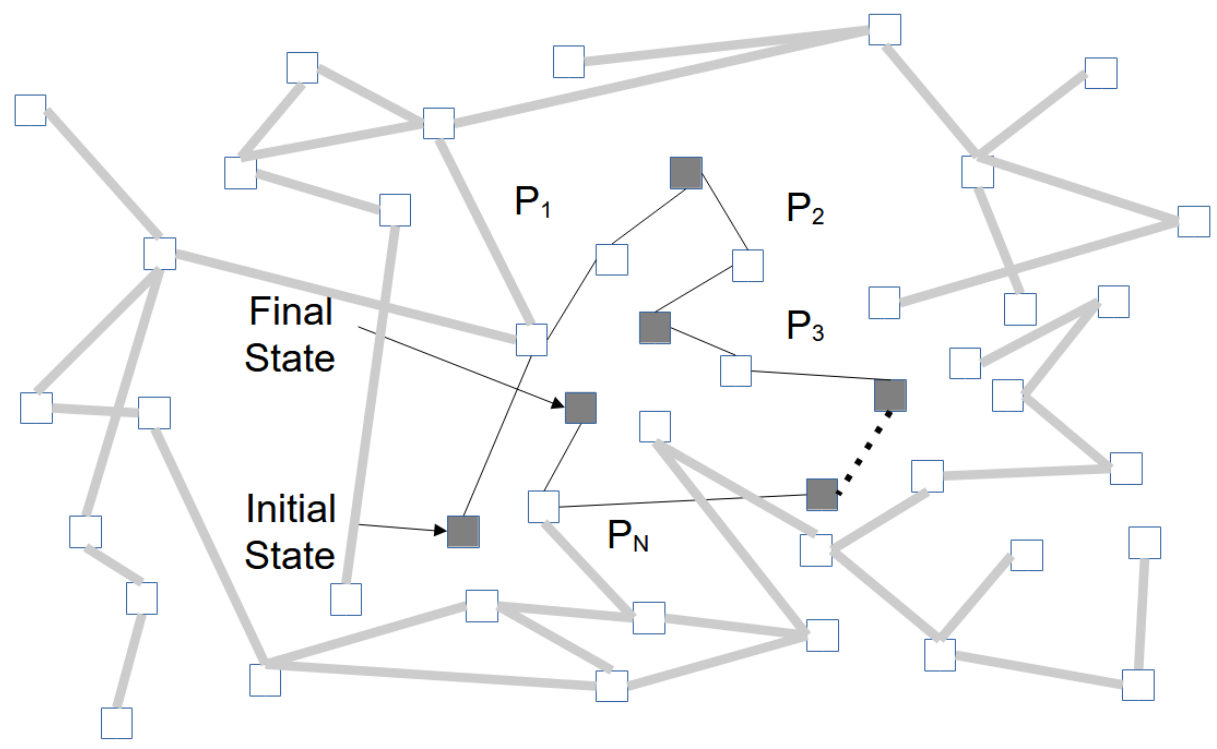

Figure 4: An indirect interaction as a path through the network of functional states represents type 2 (rational methodical) reasoning in conceptual space. 


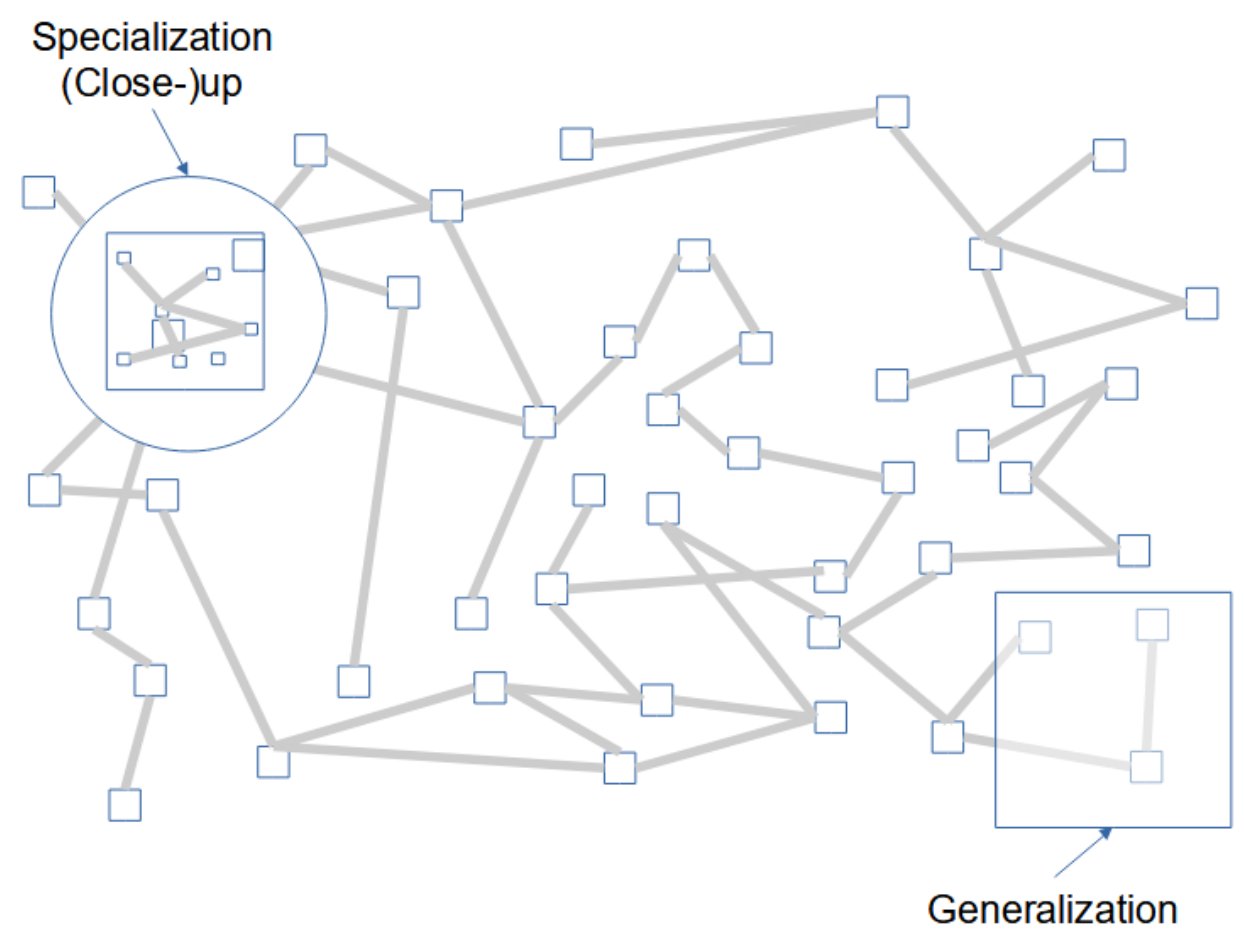

Figure 5: In functional state space the scale of nodes is not fixed. In the conceptual space that acts as the functional state space of the cognitive system, concepts might interact with generalizations of concepts, which are larger regions containing multiple smaller concepts. Specification identifies smaller concepts (see close-up) within current concepts.

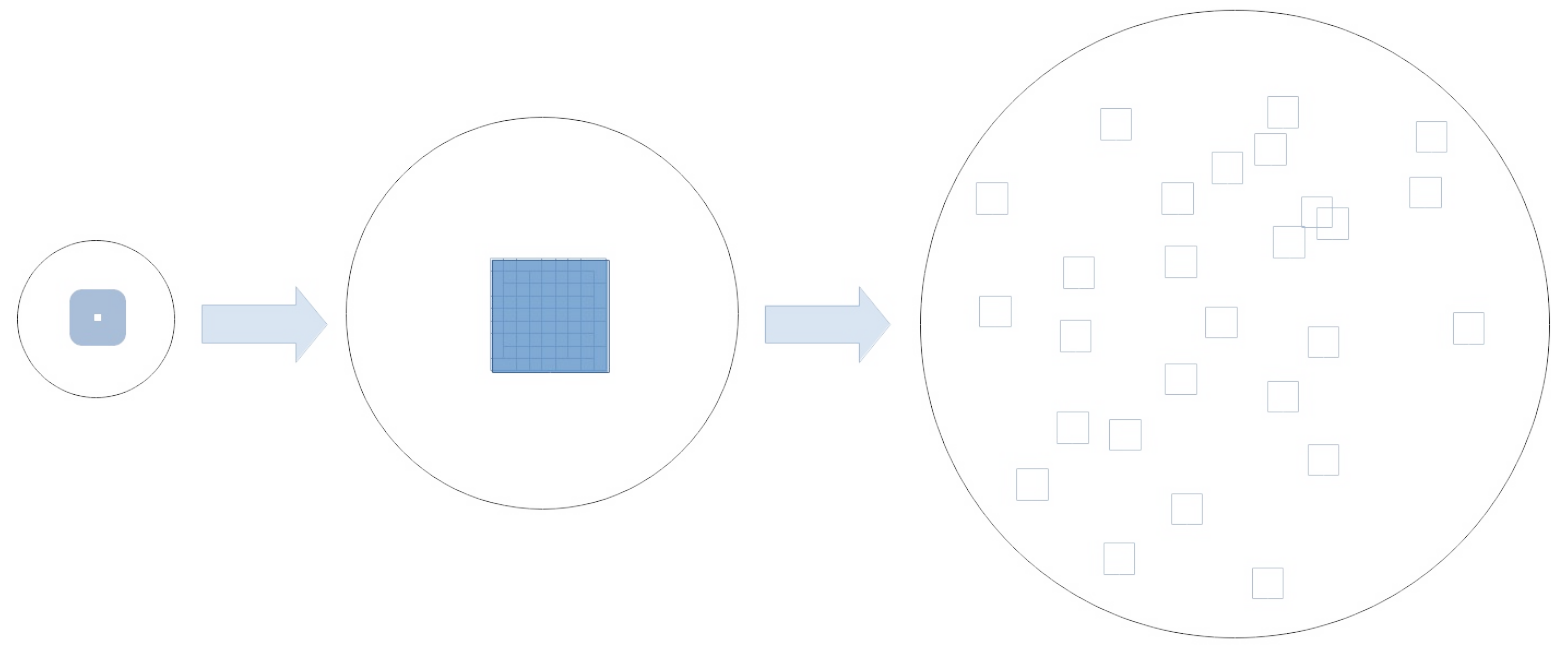

Unresolvable at Low Resolution
Structure at Higher Resolution
Resolvable

Figure 6: Unresolvable concepts at different resolutions of the conceptual space. 
As the system moves through its functional state space, it also moves through a "fitness space" that reflects its fitness to execute all of it's functions. For living systems this fitness must stay within a bounded region. Any living system can then potentially be represented by an algorithm that moves through the functional state space of that system while also moving through the fitness space of that system in a way that maintains stability within that bounded region of fitness space. Since mathematical equations do exist that form strange attractors which confine their dynamics to a bounded region, such algorithms have been theorized [2].

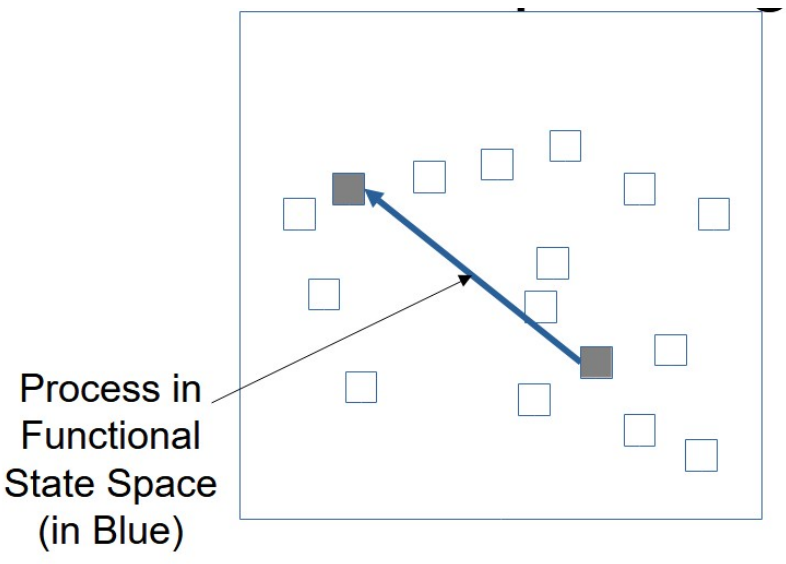

Functional State Space

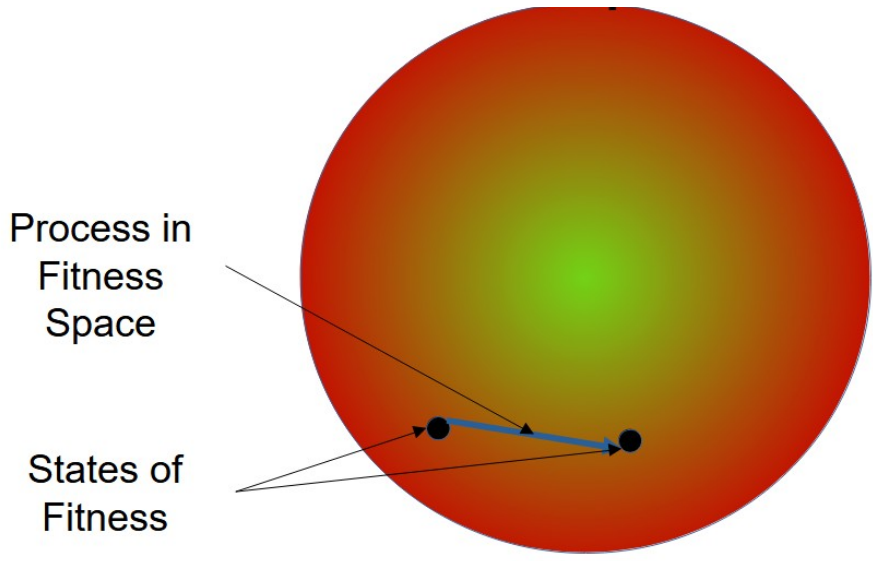

Stable Region of Fitness Space

Figure 7: Path of System in Functional State Space and Corresponding Path in Fitness Space: Cognition can be represented as being a localized system in which reasoning processes trace a path through the functional state space of the cognitive system, while also tracing a corresponding path through the fitness space of the cognitive system.

\section{$4 \quad$ Properties of Functional State Spaces}

If properties of a system can be defined in terms of the functional state space of that system, that definition can potentially be reused in other functional state spaces to determine the properties of others systems represented by those functional state spaces as well.

Definition of a System: From the perspective of Human-Centric Functional Modeling a system is defined as an entity having behaviors that start with or end in functional states that are confined to the domain object of some functional state space. For example, the domain object of conceptual space is the concept. Every reasoning process begins with or ends in a concept. Cognition is defined as the system whose functional states are confined to concepts, and whose behavior is confined to conceptual space.

General Problem-Solving Ability: For cognition, general problem-solving ability is hypothesized to be the volume of conceptual space that can be navigated per unit time, multiplied by the density of that conceptual space, and is known as intelligence. In other functional domains general problem-solving ability represents the ability of the system to get from any functional state in general to any other, and is hypothesized to be given by the volume of functional state space that can be navigated per unit time, multiplied by the density of that functional state space space.

Solution Complexity: In conceptual space hypothesized to be the length of the solution path, multiplied by the linear density of concepts along that path. In other functional domains solution 
complexity is hypothesized to represent the ability of the system to get from one specific functional state to another, and is hypothesized to be the length of the solution path in conceptual space, multiplied by the linear density of concepts along that path.

Problem Complexity: Hypothesized to be the complexity of the least complex solution required to solve the problem.

System Complexity: Represents the number of possible solutions with which the system might solve the problem of navigating from one functional state to another, and the complexity of those solutions. Hypothesized to be the volume of the functional state space multiplied by the density of the functional state space. Other work has attempted to determine a measure of complexity of a system through analyzing networks likely equivalent to the system's functional state space [9]. This has resulted in a proposed formal definition of Natural Complexity $(\mathrm{NaC})$ as a multivariable function that is intended to resolve the issue that currently every discipline "might potentially have its definition of Complexity" [9]. By contrast this definition of $\mathrm{NaC}$ is cross-disciplinary in that it might be applied in distinct disciplines. This formal definition of natural system complexity $(\mathrm{NaC})$ has yet to be compared with the hypothetical human-centric representation of system complexity.

One aspect of complexity might be referred to as Descriptive Complexity [9]. The Descriptive Complexity is related to the total information needed to describe any Complex System and its variable patterns. Part of Descriptive Complexity is Effective Complexity which depends on the level of detail, at which the entity is described. Descriptive complexity represents the complexity of describing the entire functional state space. Consistent with the idea of effective complexity, any functional state space might be described at higher or lower resolution.

It has been suggested that Complex Systems are "scale-free networks" [9], and that "living beings and their societies are Complex Adaptive Systems" [4]. In Human-Centric Functional Modeling the term "network" describes complex systems in multiple ways. Complex systems are represented as navigating the networks that describe their functional state spaces, and complex systems are represented as consisting of a network of functional components. In Human-Centric Functional Modeling systems are described as being complex systems when their functional state spaces contain complex paths which they might navigate. At this point it cannot be confirmed whether HumanCentric Functional Modeling does not in any immediately obvious way determine whether the functional state space of a complex system should or should not consist of a scale-free network. Whether HCFM is consistent with the statement that complex systems are scale-free networks remains to be explored.

\section{$5 \quad$ Exponentially Increasing General Problem-Solving Ability}

From the perspective of HCFM there are at least three ways to exponentially increase general problemsolving ability within a given domain.

\subsection{Increasing General Problem-Solving Ability by Combining into an Organism}

Human-Centric Functional Modeling represents organisms as being comprised of a hierarchy of adaptive problem-solving domains (homeostasis, cellular level autopoiesis, etc.), each of which potentially replicates the same algorithm for maintaining stability in fitness space while navigating it's respective functional state space. Some of these domains represent systems that are stand alone. Other domains represent systems that are "processes" that might occur in those stand alone systems. The importance of decoupling organisms into this hierarchy of subsystems, each of which consists of a 
number of functional components, is that as calculated in the supplementary data to this paper, a system with $\mathrm{N}$ functional components can have exponentially greater general problem-solving ability and hence exponentially greater capacity to manage complexity than an ecosystem of $\mathrm{N}$ individual entities. Human-Centric Functional Modeling is hypothesized to take exponential problems with large $\mathrm{N}$ that are intractable even with modern supercomputers [9], and decouple them into different adaptive domains so they might become transformed in Non-deterministic Polynomial problems or NPproblems that are both solvable and tractable.

Even the simplest forms of life are believed to be complex systems [9], and therefore consist of exponential problems. In order to be able to execute the normal functions of life, they must be able to reliably solve those exponential problems. If the solutions that nature has developed to solve those problems are generally reused, it might be expected to see those solutions throughout natural systems.

\subsection{Increasing the General Problem-Solving Ability of the Organism}

In conceptual space, once the ability to generalize spans the entire conceptual space, the conceptual space can undergo an exponential increase in size and density, representing an exponential increase in potential for general problem-solving ability (intelligence) [5].

Since there are no physical limits to the size of an AGI, in order to achieve this exponential increase in intelligence in an AGI additional functional components might be added to grow that AGI and to grow its intelligence as required. That AGI must introduce the ability for one individual functional component to find information and reasoning within the conceptual space of another individual functional component. Similarly, in order to achieve this exponential increase in intelligence in a GCI, that GCI must introduce the ability for one individual to find information and reasoning within the conceptual space of another individual so that it is possible to exponentially increase the number of involved in the GCI. In order for that to happen, the AGI or GCI must introduce the capacity to generalize as required for one individual functional component, or one individual human, to locate information in the conceptual space of another.

\subsection{Increasing Capacity for Cooperation}

If problem-solving is decentralized then the process of problem-solving must be able to be initiated by any single functional component in the system. If the resource needs of the $\mathrm{N}$ functional components involved in the problem-solving process is larger than the resources available from that single functional component initiating that process, then without addressing that gap a problem-solving process with massive $\mathrm{N}$ cannot be executed. For this reason, in addition to having general problemsolving ability in the cognitive domain, the cognitive system is represented as having general problemsolving ability in the "cooperation domain" which is intended to represent the domain of functionality that allows cells to self-assemble in a self-sustaining way into massive networks of multi-cellular cooperation of the size required. That is, a domain able to adapt to problems of cooperation. In the functional model of cognition this cooperation domain assembles cooperation between the functional components of the cognitive system rather than between single cells. However, since the functional model of the cognitive system makes no determination of how those functional components are implemented, they could very well be single neural or other cells as well. The first principle involved in the cooperation domain is hypothesized to be that where outcomes can be scaled with cooperation, the scale of cooperation can be increased by executing processes in series and in parallel, until outcomes are increased to a sufficient degree. 

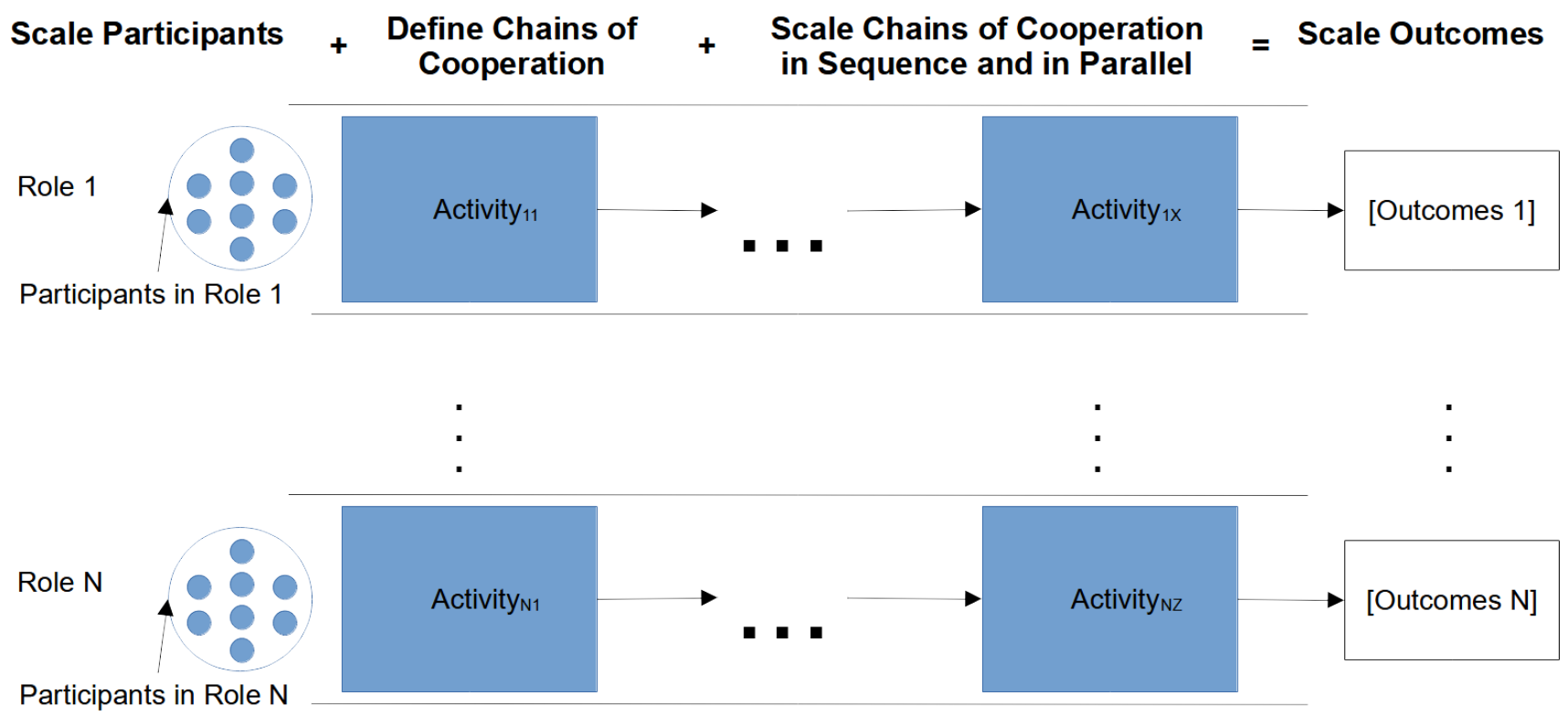

Figure 8: Patterns of cooperation.

The second principle involved in this domain is that the functional component initiating a process must not only incentivize other functional components to participate in that process, but must also incentivize those functional components to recruit others, and must do so in a self-sustaining way so that cooperation can be scaled massively.

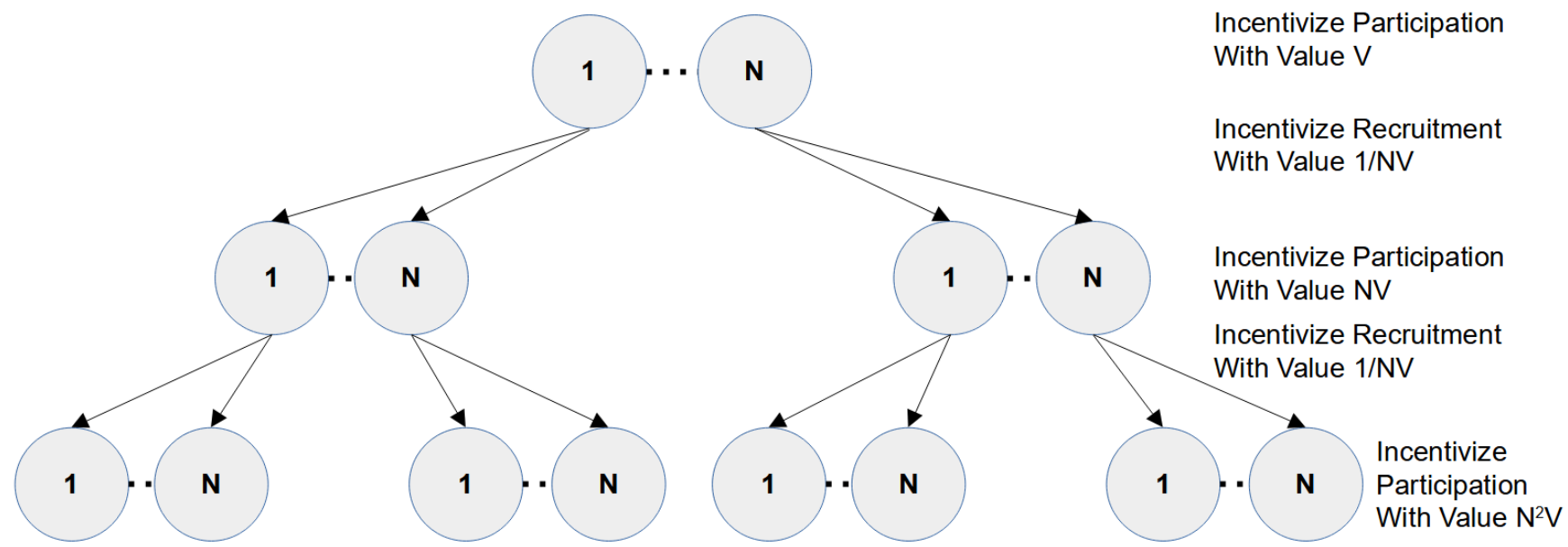

Figure 9: Initiating execution of a collective process by an individual functional component of a system.

The principles in the cooperation domain have not yet been elaborated into a set of abstract functions that span this functional state space. However, their practical implications have been explored in the design of software platforms intended to organize crowds at massive scale [11]. 


\subsection{Application to Sustainable Development}

Value chains that leverage collective intelligence based cooperation have been shown to have the potential to exponentially increase impact on whatever collective social, economic, environmental or other challenge has been targeted, such as in the case of increasing agricultural livelihoods per program dollar [12]. Current working papers explore how a ten phase Collective Intelligence based Program to Accelerate Achievement of the Sustainable Development Goals (CIPAA-SDGs) based on HumanCentric Functional Modeling might be used to exponentially increase sustainable development in a wide number of other areas so that it becomes sustainably self-funding and can occur at the scale required to transform communities globally. In addition to exploring how an exponential increase in outcomes might be achieved in agriculture, various working papers explore how exponential increases in outcomes might also be achieve in education, healthcare, employment, and sustainable housing development.

While an exponential increase in ability to solve a narrow problem might be achievable today [8], achieving an exponential increase in ability to solve any problem in general, and doing so in a way that reliably serves the collective good likely requires a system that optimizes collective good such as GCI. Therefore, introducing GCI and its capacity for an exponential increase in collective outcomes is likely required to make sustainable development reliably achievable.

Any collective problem that has not yet been solved might not be reliably solvable without this exponential increase in intelligence believed to accompany GCI. While ability to solve a specific problem might reliably be exponentially increased [8], that narrow problem-solving ability cannot be guaranteed to be directed towards the correct problem as required to exponentially increase collective outcomes. For this an exponential increase in general problem-solving ability might be required. In summary, according to the model and contrary to the opinions expressed by others [9], existential challenges like achieving green growth, achieving a circular economy, or solving climate change, might not be reliably solvable without GCI, and might in fact be reliably unsolvable. This statement, which has been expressed in various forms by others [13], is in itself is a profoundly controversial statement since trillions of dollars are currently invested in the ideology that the SDGs can be achieved if more money is dedicated to the same approaches currently being used. General Collective Intelligence introduces the possibility of evaluating the fitness of an exponentially larger number of value chains of far greater complexity. GCI also introduces the possibility of evaluating the fitness of an exponentially larger number of processes to address problems in executing those value chains, thereby potentially making execution far more reliable and robust than achievable today.

Once this cooperation has been discovered, it must be able to be stored so that the complexity of the patterns can continue to evolve, enabling their impact to continue to be increased. The concept of a genome storing these networks of cooperation between billions or more entities also might require GCI [21]. Technology removes the limits to consumption, permitting exponentially higher rates of consumption than is possible in the natural world. In order to make sustainability reliably achievable, it is reasonable that capacity for cooperation must be scaled exponentially as well.

Once all sustainable development interventions can be represented as paths through the collective conceptual space, then all possible interventions can be compared, and the same general patterns identifying the best solutions can be applied to exponentially increase that impact [14] to the point that impact is sustainably self-funding at the scale required to transform the world. This is part of a general pattern as shown in table 1 in which Human-Centric Functional Modeling is used to decouple knowledge within a given domain into functional components that might be added to a library, so that 
cognitive technology might leverage that library to converge on the single most "fit" model at solving any general problem in that domain, thereby creating the possibility of exponentially increasing problem-solving ability in that domain.

\section{Domain}

Psychology

Sustainable Development

Future Internet

Blockchain

Artificial General Intelligence

General Collective Intelligence

Systems Thinking

\section{Use of Human-Centric Functional Modeling}

Use cognitive technology to maximize ability to converge on the single most "fit" model of psychology in each context of use (to be discussed in the upcoming workshop).

The CIPAA-SDGs program leverages GCI to gain the capacity to exponentially increase social, economic, environmental, or other collective impact. [14]

Use cognitive technology to maximize ability to converge on the single most "fit" model of the Future Internet. [15]

Use cognitive technology to maximize ability to converge on the single most "fit" model for blockchain platforms. [16]

Decouple Artificial Intelligence (AI) functionality into a library that maximizes ability to implement AGI. [17]

Decouple Collective Intelligence (CI) functionality into a library that maximizes ability to implement GCI. [18]

Use HCFM to model systems thinking theories in order to suggest a theoretical validation for a meta theory in systems thinking [20]

Table 1: Pattern in the use of Human-Centric Functional Modeling in different problem domains.

\subsection{Application to Physics}

Modeling the physical universe semantically creates the possibility of storing all data from all theoretical or experimental measurements in the same data model. Modeling all theories semantically creates the possibility of testing every component of every theory against every other one and against all available data to more reliably converge on the single understanding that is most fit in describing the observed function of the universe. 

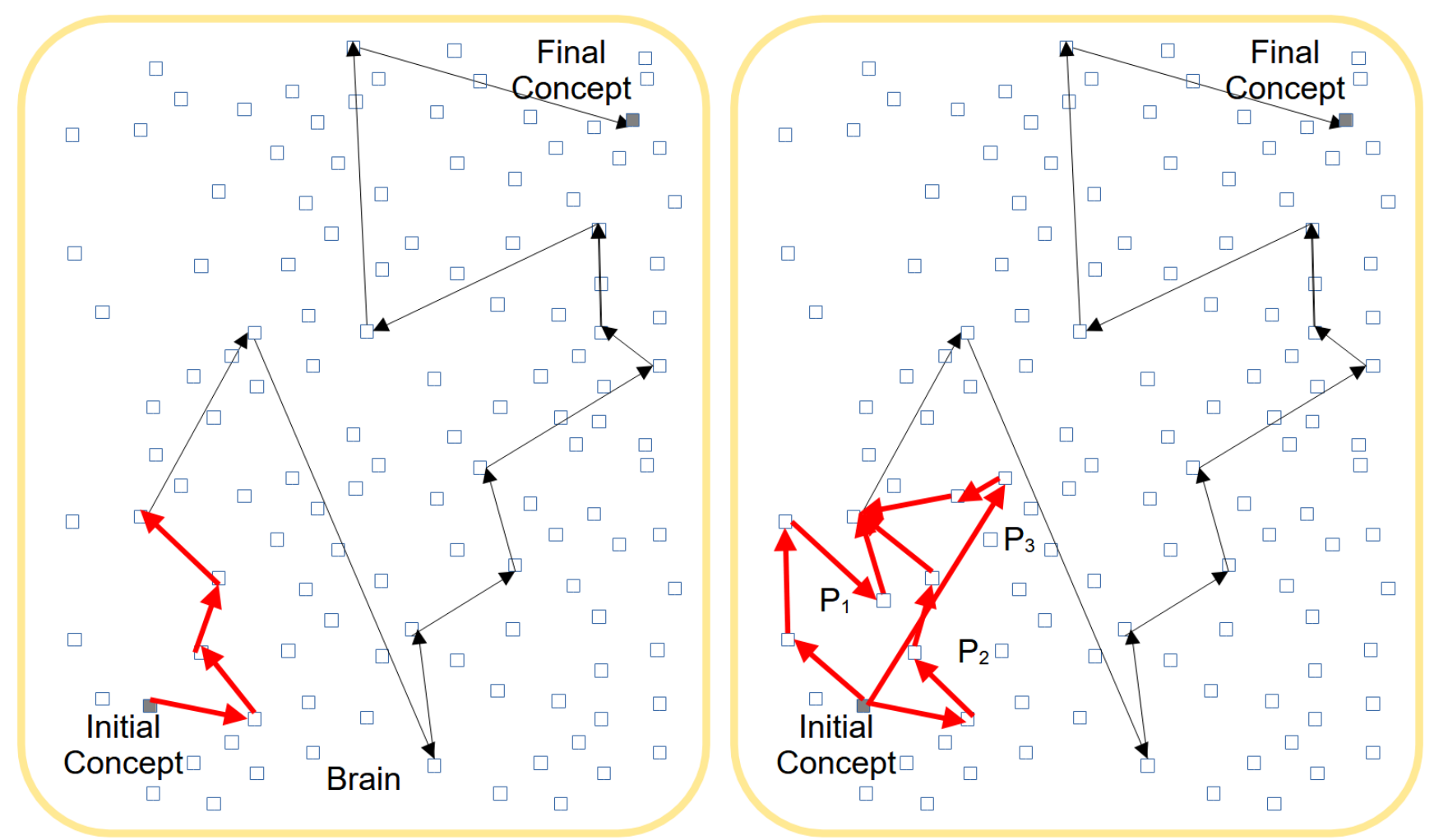

Figure 10: Left: Theory with initial reasoning segment in red. Right: Theory with selected reasoning replaced by components of other theories.

With a semantic data model every simulation, every computer game, every CAD design, every Computer Generated Image (CGI), or every other computer interaction with a real or imaginary world becomes part of a single dataset that can potentially be leveraged by every other such computer interaction. This means that any representation or animation of any imaginary world, or of the real physical world, is stored in the same way and can potentially be reused in any game, any simulation, any CAD application, any CGI animation, or anything else for any purpose. The ability to decouple every component of every model so that it can potentially be reused in every other production, simulation, or game, combined with semantic search to identify those opportunities for reuse, and combined with General Collective Intelligence to multiply those opportunities, is expected to exponentially increase the market for such intellectual property. In this way, every game might contribute simulation or other infrastructure that might be used in all physics research and vice versa, thereby effectively multiplying research funding for both. 


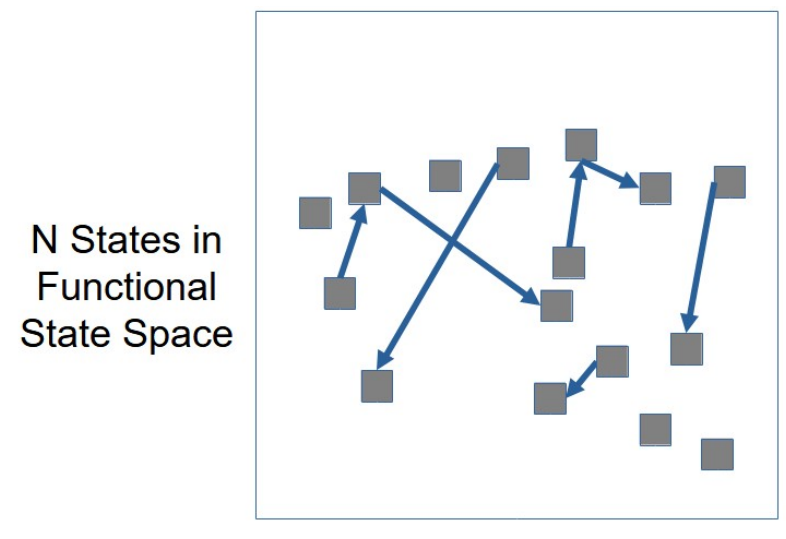

Functional State Space

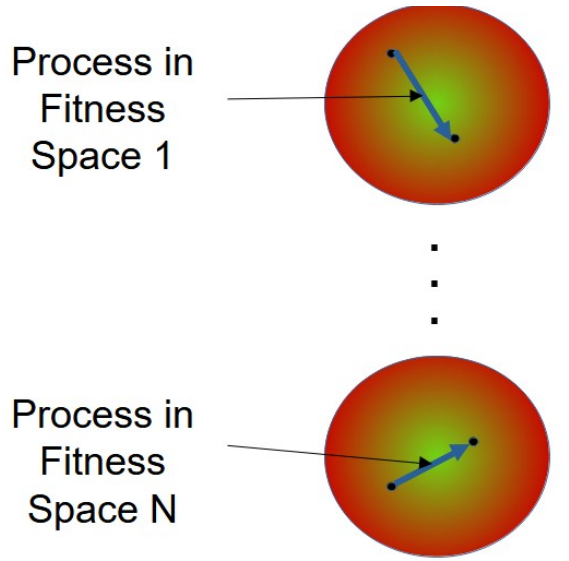

Stable Region of Each Fitness Space "i"

Figure 11: Paths of a Distributed System in Functional State Space and Corresponding Paths in Fitness Spaces: The physical universe can be represented as a distributed system in which each point in functional state space evolves according to its motion in it's own fitness space. The evolution of a distributed system such as the physical universe might reuse tools created to simulate the evolution of a localized system such as cognition, and vice versa.

Because functional state spaces naturally undergo a phase change resulting in a "big bang" when the distance over which functional states might interact spans the entire functional state space, and because this phase change in functional state space is repeating, modeling the physical universe as a functional state space has the potential to reveal new insights about the big bang, including the conditions under which it might be cyclical. Furthermore, functional state spaces can be used to represent universes with different physical laws. Other research has explored how our universe can potentially be constructed as a hierarchy of much simpler universes and the interactions between them [6]. Modeling all universes semantically allows new relationships between those universes to be discovered through executing well-defined operations (translation, rotation, reflection, etc.) on existing universes that might produce new universes through their interactions. This might provide the opportunity to gain new insights about parts of our universe such as dark matter that we don't appear to interact with, as well as filling other gaps in our understanding of the universe. It remains to be explored how the most commonly accepted theories in physics might be reinterpreted using HCFM, and why doing so is useful, so this might be communicated in more detail to a larger and more specialized audience of physicists in a workshop that might identify the most important areas of research required to validate each application of HCFM in each area of specialization.

\subsection{Application to Evolutionary Biology}

Human-Centric Functional Modeling can be used to define organisms as a hierarchy of systems, all of which are implemented by some functional component that in turn is an implementation of the same generalized algorithm that living processes can be represented as using to achieve adaptive problemsolving ability. Every model of every biological process can potentially be viewed this way. By decoupling the components of every biological process model into these functional components, all biological models can be added to a library that a General Collective Intelligence might use to maximize our collective ability to come to the single understanding that is most fit in representing the observed function of all processes in biological systems. As with physics it remains to be explored how 
the most common biological models might be decomposed this way using HCFM, and why doing so is useful, so this might be communicated in more detail to a larger audience of biologists in a workshop that might identify the most important areas of research required to validate these ideas.

\subsection{Application to Psychology}

Various meta-theories of psychology exist, but the challenge is that the problem of deciding which one is the best is an intractable problem. Human-Centric Functional Modeling can potentially be used to define all of the functions of human cognition and can be used to describe all of the concepts as well as all the reasoning and understanding processes it contains. Every branch of psychology likely has a very well defined meaning in conceptual space. For example, developmental psychology might represent the formation of conceptual space, addiction psychology might represent the presence of certain thought patterns in conceptual space. Whatever the case, every intervention that might be achieved through any theory in any branch of psychology represents a path through conceptual space. Using General Collective Intelligence it might be possible to exponentially increase our collective capacity to detect some set of interventions that achieve exponentially greater outcomes per input of resources, thereby creating the possibility of reliably converging on the single understanding of psychology that is most "fit".

\subsection{Application to Computer Science and Engineering and Blockchain Technology}

Current papers (table 1) explore how "cognitive computing" based on Human-Centric Functional Modeling might be used to exponentially increase outcomes of computing, and how the "cognitive blockchain" based on Human-Centric Functional Modeling might be used to exponentially increase outcomes achieved by blockchain platforms.

\section{Future Directions: Potential Workshop}

It would be useful to collaborate with individuals in these disciplines (physics and mathematics, evolutionary biology, psychology, computer science and engineering, blockchain technology, and sustainable development) to deliver a workshop that explores how Human-Centric Functional Modeling might exponentially increase research impact in these disciplines, but that conducts this exploration in a way that decouples keynote speakers at that workshop from requiring expertise in HCFM or in any other areas outside their own discipline. Current plans are to have keynote speakers in each of these disciplines identify what's the most important impact on their discipline that they are looking to use systems thinking to achieve and what are the barriers to achieving it. Working with experts in Human-Centric Functional Modeling and General Collective Intelligence that impact might be quantified and mechanisms might be suggested that are predicted to enable that impact to be exponentially increased. The keynote speaker or speakers in each disciple might then explore the validity of these suggestions and educate their peers with their findings. After this keynote speakers might host a discussion to explore the validity of their own suggestions in any way that they as the host sees fit.

\section{$7 \quad$ Larger Implications}

It has been suggested that limitations in scientific predictability make many cutting-edge technologies highly disputable and raise ethical issues because we simply don't know what the impact will be [9]. However, from the perspective of Human-Centric Functional Modeling, that which is ethical is 
synonymous with that which is good, and good is defined as that which serves to increase the fitness of life collectively. Nature appears to have solved the problem of developing ethically in that whatever entities it has evolved over the course of billions of years has so far continued to nurture life. However, it has also been stated that real complex networks are difficult to study [22], [23], [24], [25], and it has been stated that "accurate structural, functional, and dynamical analyses of any Complex System are often challenging tasks" [26]. In contrast, by using Human-Centric Functional Modeling to define representations of complex systems, and by assuming that the behavior of those systems must stay within certain boundary conditions, outcomes related to those boundary conditions might become highly predictable. As one example, it has been mentioned previously that HCFM has been used to define a model for an Artificial General Intelligence with the potential for exponentially greater general problem-solving ability that might be directed at any problem by any individual. HCFM has also been used to define a model of General Collective Intelligence with the potential for exponentially greater general problem-solving ability and therefore exponentially greater ability to achieve impact on any collective problem. An AGI is represented as optimizing outcomes for some individual entity, whereas a GCI is represented as optimizing collective outcomes. Which particular outcome will be targeted for optimization by an individual or group is uncertain, but what can be known is that the more powerful the AGI the more powerful the optimization, and the more powerful the optimization for a given individual, the more powerful the accumulation of competitive advantage and the accumulation of resources accompanying that competitive advantage. The only possible outcome of unequal accumulation of resources is increased inequality between the upper and lower segments of the population, though the potential impact on raising or lowering the resource levels of the median of the population are uncertain.

Neither of these exponential increases in general problem-solving ability are hypothesized to have been possible before or to be possible otherwise. These increases are thus the most important potential sources of impact, but each has an impact in the opposite direction. Where AGI acts as a system of individual optimization, a GCI acts as a system of collective optimization. Therefore Human-Centric Functional Modeling suggests that the most important element in scientific predictability is whether decision-making continues to act as a system of individual optimization that optimizes outcomes for some entity, or whether decision-making comes to act as a system of collective optimization that optimizes outcomes for civilization as a whole.

It has been stated that "if the final goal is the control of the Complex System behavior, it could be enough to identify the set of driver nodes that can guide the system's entire dynamics" [27]. In functional state space behaviors are paths from a node representing one functional state to a node representing another functional state. Certain nodes might be highly likely to result in given behaviors. Therefore looking for driver nodes in the network of functional states might be equivalent to looking for behaviors constrained by existing boundary conditions such as above. It has also been stated with respect to Complex Systems that "science finds insurmountable obstacles in predicting their behavior, especially in the long term" [9]. However, with HCFM capacity for complexity does appear to be predictable as in figure 12. Once HCFM has been used to develop cognitive computing systems with greater capacity for complexity as in figure 13, far greater predictive ability might be achievable. 


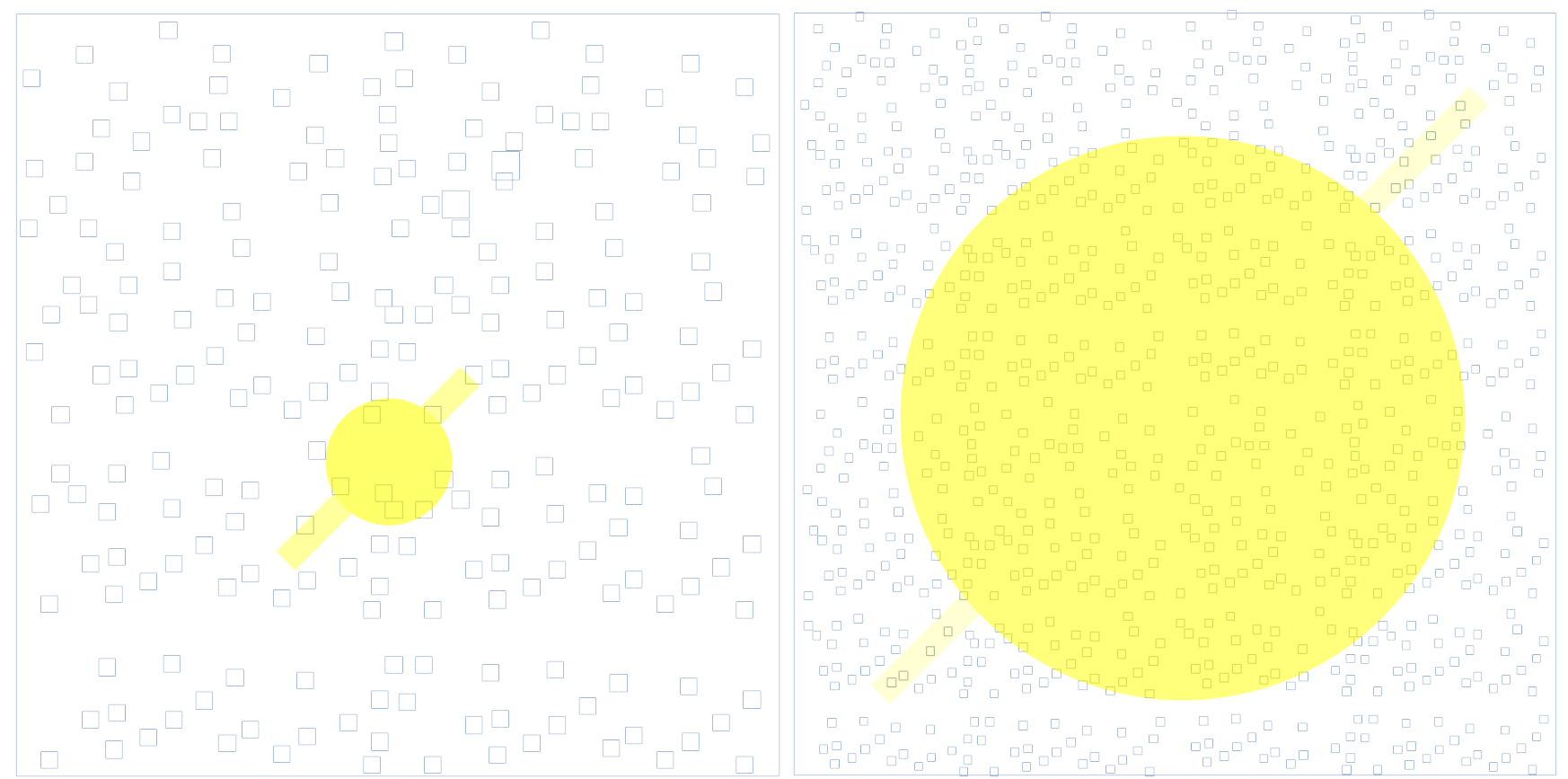

Figure 12: Left: The yellow sphere represents the conceptual space accessible with human cognition. The yellow rectangle represents the increased narrow problem-solving ability achievable through external computing tools. Right: The larger yellow sphere on a more dense conceptual space represents the exponentially larger and denser conceptual space accessible with cognitive computing. The yellow rectangle represents exponentially more powerful external computing tools.

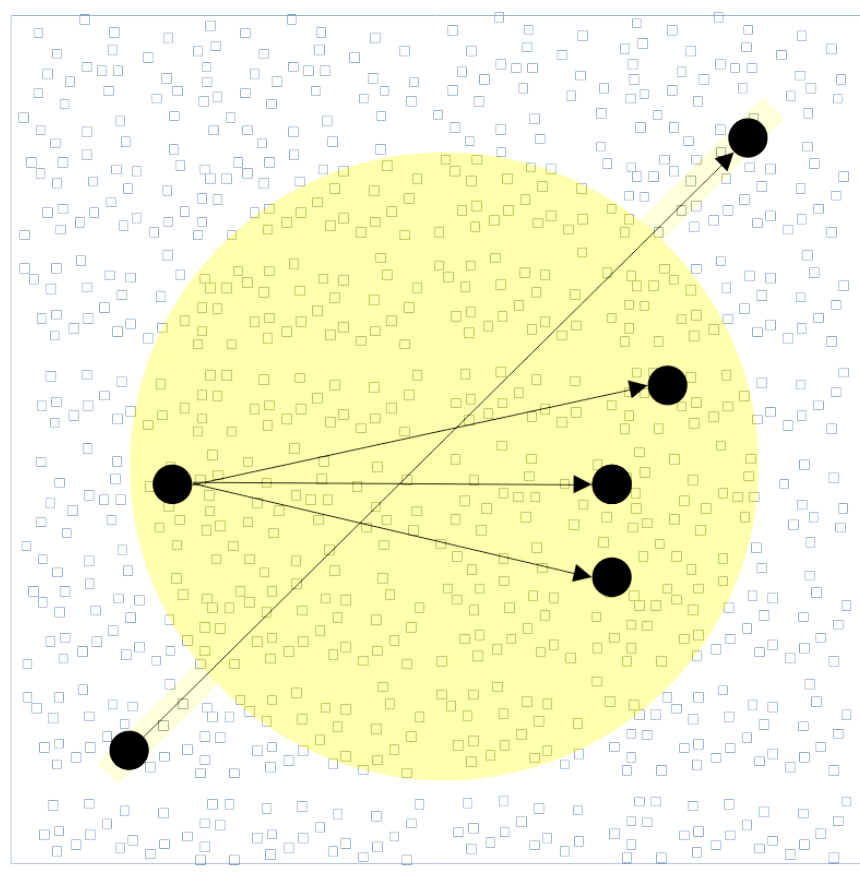

Figure 13: The gap between two black dots represents a problem. The arrow between them represents a solution. When the solution can be anywhere within the sphere flexibility is required and cognitive computing is advantageous. Then the solution is within the rectangle computing power is required and traditional computing is advantageous. 
According to Gentili [9], the cognitive scientists Gallistel and King [31] and the neuroscientist Marr [32] have proposed a methodology to describe and understand any Complex System through three levels of analysis. The first analysis at the "computational level" describes processes in the system as computations on inputs and outputs. The second analysis at the "algorithmic level" consists of formulating algorithms that might carry out those computations. The third and conclusive analysis is at the "implementation level" and consists of looking for mechanisms to implement the algorithms. If the analyses at the three levels have been conducted properly, the mechanism found in the final analysis is expected by those scientists to be a plausible replication of the Complex System's behavior. According to Gentili discovery of a systematic way to leverage such a methodology is hoped to allow us to better understand Complex Systems and is expected to provide new tools to reach the goals of the 2030 Agenda. It is also suggested to be likely to promote the formulation of a brand new transdisciplinary theory concerning Natural Complexity [33], [34]. It is expected that all of this will likely require representing the human meaning of data, that is, representing data semantically. Human-Centric Functional Modeling might address all the requirements for that systematic method. Analysis at the computational level is equivalent to describing the functional state space of the system. Analysis at the algorithmic level is equivalent to defining an algorithm which the system might use to navigate its functional state space. Analysis at the implementation level is equivalent to combining all implementations into a library that such an algorithm might use to navigate that functional state space, and in doing so replicating the behavior of the system. Finally HCFM potentially provides the first complete semantic representation of meaning so that for the first time data might be modeled semantically.

The ten phase CIPAA-SDGs program discussed here demonstrates the power or HCFM through leveraging a model of collective cognition (General Collective Intelligence or GCI) developed using Human-Centric Functional Modeling to potentially gain the capacity to self-assemble into a network of projects that is sustainably self-funding at the multi-trillion dollar scale required to transform communities globally. However, in order to build the massive mind share required to launch such a project, it's important to build a network of researchers who can explain these concepts to the community of people in their own specialist discipline and who can build teams and secure research funding to elaborate these concepts and how they apply in their fields. In order to elaborate the concepts and to develop the technologies required to implement this program, a set of Large Scale international Collaborative REsearch (LSCORE) initiatives have been designed to engage the international research community from across a wide range of disciplines to do so, since being based on a common functional model, every theoretical component elaborated and any technology component implemented for any discipline, might solve the problem of doing so in a way that can be reused across all disciplines. Because of this potential to multiply impact, in providing the opportunity to achieve this multiplication HCFM might be the most important systems movement today.

\section{Conclusions}

Human-Centric Functional Modeling, combined with the model of Artificial General Intelligence and the model of General Collective Intelligence it has been used to define, are hypothesized to be innovations that are disruptive on an unprecedented scale. According to one living mathematician "if the NP were reduced to P problems, humanity would become able to understand nature as never before" [9]. According to Kurt Gödel the discovery that the NPs are reducible to P problems would have "consequences of the greatest magnitude" [28]. One of those predicted consequences is that human civilizations would be transformed to become radically more efficient at achieving outcomes [29], which would be expected to dramatically impact capacity to achieve the SDGs. 
The final goal of Complexity Science is trans-disciplinarity, which will be achieved when Complexity Science formulates a uniform description of Complex Systems, breaking down the traditional disciplinary boundaries. Through defining all systems in terms of innate human perception, HumanCentric Functional Modeling potentially provides a uniform description of Complex Systems that breaks down the traditional disciplinary boundaries in this way. It has been suggested that interdisciplinary efforts spanning all disciplines are required to tackle the Complexity Challenges [9], and that investigation of Complex Systems demands the collection, storage, and elaboration of massive datasets or so-called Big Data [30]. Using the example of natural organisms and their construction in terms of components such as cells, through organizing the decentralized collection, storage, and processing of datasets through a massive number of entities, the capacity to collectively navigate enormous streams of data might significantly increase capacity to investigate complex systems until doing so is reliably achievable. With respect to trans-disciplinarity, from the perspective of HumanCentric Functional Modeling, defining any problem and discovering any solution in any discipline helps specify the nodes and edges in some region of conceptual space, so those problem definitions and solutions might be generalized and reused in any other discipline (any other region of conceptual space) where they apply.

In any high level summary such as this, without a detailed question from each individual audience member it's impossible to describe in detail the specific potential application that individual might need to have described for them in order to understand the concept. The more disruptive the innovation is the more problems it might impact, and the higher the level of abstraction that might initially be needed to communicate it's relevance to all prospective listeners. Despite the lack of a sufficiently detailed description in the initial high-level summary to conclusively demonstrate the validity of these claims for all its audience, through following up by asking what the implications might be in each discipline so those implications might be tested, it is still possible to reliably progress in an incremental way towards communicating an understanding of disruptive innovations. Being able to encourage sufficient feedback to hold an interdisciplinary workshop is an excellent start. This avoids setting the criteria for proceeding as being that the first initial summary has answered all questions required to conclusively validate it's claims from the perspective of each and every individual who might be in the prospective audience. Peer-review and other processes that effectively amount to such criteria might frequently be introduced inadvertently if not carefully guarded against, with the result that all communication about sufficiently disruptive innovations must reliably fail, and sufficiently revolutionary advances in systems thinking can never be propagated.

\section{References}

[1] Newman MEJ (2011) Complex systems: a survey. Am J Phys 79:800-810. https://doi.org/10.1119/1.3590372

[2] Andy E. Williams, A Model for Artificial General Intelligence, Chapter, Artificial General Intelligence, Series: Lecture Notes in Computer Science, Year: 2020, Volume 12177, Pages 357-369, DOI: $10.1007 / 978-3-030-52152-3 \_38$

[3] Williams, A. E., Defining a Continuum from Individual, to Swarm, to Collective Intelligence, to General Collective Intelligence, International Journal of Collaborative Intelligence, in press (2021)

[4] Miller JH, Page SE (2007) Complex adaptive systems An introduction to computational models of social life. Princeton University Press, Princeton 
[5] Williams, A. E., Dos Santos, Monika, Haliki, Emir (2020, July 11). Human Intelligence and General Collective Intelligence as Phase Changes in Animal Intelligence. https://doi.org/10.31234/osf.io/dr8qn

[6] Stephen Wolfram, A New Kind of Science, 2002, Wolfram Media, Champaign, IL, ISBN: 1-57955008-8

[7] Newman MEJ (2010) Networks: an introduction. Oxford University Press, New York

[8] Williams, A. E., Cognitive Computing and its Relationship to Computing Methods and Advanced Computing from a Human-Centric Functional Modeling Perspective, Soft Computing Research Society SCRS Conference Proceedings on Intelligent Systems” (Open Access | ISBN 978-93-91842-08-6), accepted (2021)

[9] Gentili, P.L. Why is Complexity Science valuable for reaching the goals of the UN 2030 Agenda?. Rend. Fis. Acc. Lincei 32, 117-134 (2021). https://doi.org/10.1007/s12210-020-00972-0

[10] Stahel WR (2016) Circular economy. Nature 531:435-438. https://doi.org/10.1038/531435a

[11] Williams, A. E., Approximating an Artificial General Intelligence or a General Collective Intelligence, International Journal of Collaborative Intelligence, in press (2021)

[12] Williams, A. E. (2020, April 30). The Collective Intelligence based Program to Accelerate Achievement of the Sustainable Development Goals as a Case Study for Collectively Intelligent Program Design. https://oi.org/10.31235/osf.io/r2dxq

[13] Hickel, Jason, and Giorgos Kallis. "Is green growth possible?." New political economy 25.4 (2020): 469-486.

[14] Williams, A. E. (2020, April 30). The Collective Intelligence based Program to Accelerate Achievement of the Sustainable Development Goals as a Case Study for Collectively Intelligent Program Design. https://oi.org/10.31235/osf.io/r2dxq

[15] Williams, A. E., \& Petrovic, N. (2021, January 12). The Role of Cognitive Computing and Collective Cognitive Computing in the Future Internet. https://oi.org/10.31730/osf.io/qb7sm

[16] Andy E. Williams, Roberto Moro Visconti, The Application of Artificial General Intelligence to the Cognitive Blockchain and the Internet of Value, December 2020, DOI:

10.13140/RG.2.2.15004.31363

[17] Williams, A. E. (2020, April 27). Defining Functional Models of Artificial Intelligence Solutions to Create a Library that an Artificial General Intelligence can use to Increase General Problem Solving Ability. https://doi.org/10.31730/osf.io/hpzb7

[18] Williams, A. E. (2020, August 19). Defining Functional Models of Collective Intelligence Solutions to Create a Library a General Collective Intelligence can use to Increase General Problem Solving Ability. https://doi.org/10.31730/osf.io/q75rv 
[19] Gregg Henriques, Andy E. Williams, General Collective Intelligence and Systemic Error in Groups, retrieved from https://www.psychologytoday.com/us/blog/theory-knowledge/202106/generalcollective-intelligence-and-systemic-error-in-groups, on July 19, 2021.

[20] Williams, Andy E. (2021b) 2021. "Human-Centric Functional Modeling and the Unification of Systems Thinking Approaches: A Short Communication”. Journal of Systems Thinking 1 (1):5. https://journalofsystemsthinking.org/index.php/jost/article/view/1369

[21] Williams, A. E. (2020, December 24). Defining the Genome and Gametes of a General Collective Intelligence Based Smart City. https://doi.org/10.31730/osf.io/b6ep8

[22] Strogatz SH (2001) Exploring complex networks. Nature 410:268-276.

https://doi.org/10.1038/35065725

[23] Barabási A-L (2014) Linked. Basic Books, New York

[24] Caldarelli G, Catanzaro M (2012) A very short introduction to networks. Oxford University Press, Oxford

[25] Caldarelli G, Vespignani A (eds) (2007) Large scale structure and dynamics of complex networks: from information technology to finance and natural science. World Scientific, Singapore

[26] Farine DR, Whitehead H (2015) Constructing, conducting and interpreting animal social network analysis. J Anim Ecol 84:1144-1163. https://doi.org/10.1111/1365-2656.12418

[27] Liu Y-Y, Slotine J-J, Barabási A-L (2013) Observability of complex systems. Proc Natl Acad Sci USA 110(7):2460-2465. https://doi.org/10.1073/pnas.1215508110

[28] Sipser M (1992) The history and status of the P versus NP question. Proc ACM STOC. https://doi.org/10.1145/129712.129771

[29] Fortnow L (2009) The status of the P versus NP problem. Commun ACM 52:78-86. https://doi.org/10.1145/1562164.1562186

[30] Marx V (2013) The big challenges of big data. Nature 498:255-260.

https://doi.org/10.1038/498255a

[31] Gallistel CR, King A (2010) Memory and the computational brain: why cognitive science will transform neuroscience. Wiley-Balckwell, New York

[32] Marr D (2010) Vision. A computational investigation into the human representation and processing of visual information. The MIT Press, Cambridge

[33] Gentili PL (2018a) Untangling complex systems: a grand challenge for science. CRC Press, Boca Raton

[34] Gentili PL (2018b) The fuzziness of the molecular world and its perspectives. Molecules 23(8):2074. https://doi.org/10.3390/molecules23082074 